\title{
Now is the time to take the next step
}

\author{
Matthieu Guémann ${ }^{1,2}$, Marius Lebret ${ }^{3}$, Clément Médrinal $^{4,5,6}$ and Thomas Rulleau ${ }^{7}$ \\ ${ }^{1}$ French Armed Forces Biomedical Research Institute, Brétigny-sur-Orge, France, ${ }^{2}$ International Society of Physiotherapy Journal Editors, ${ }^{3}$ ERFPS, \\ Physiotherapy School, Rouen, France, ${ }^{4}$ Université Paris-Saclay, UVSQ, Erphan, Versailles, France, ${ }^{5}$ Intensive Care Unit department, Le Havre Hospital,

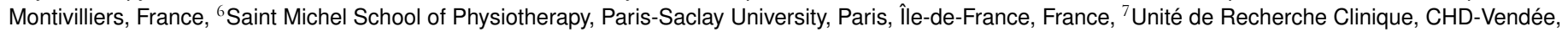 \\ La Roche Sur Yon, France
}

DOI: $10.52057 /$ erj.v1i1.6

received : 2 June 2021

accepted: 17 June 2021

W hen considering the physiotherapy landscape in France, we can observe the multitude of different colors. Our profession is built and driven by many different forces such as clinicians, scientists, educators, students, syndicates, and policy makers. All these components are essential for the growing and the independence of the profession. In this context, the position and the role of a scientific society might sometimes appear unclear, especially when defending the professional interest. However, the French Physiotherapy Society is driven by using science to serve the best interest of the profession and equally to serve the best interests of those seeking assistance from physiotherapists, patients. However, how can research be conducted in an eminently clinical field of work where vision from the scientific perspective and the clinical one often seems to move in opposite directions? On one hand, the scientist seeks to unravel mechanisms and correlations; searching for a wider context and interpreting facts relying on a population perspective to establish causation and avoid all possible bias in his/her reasoning. On the other hand, the clinician masters the art of managing situations, attaches importance to every detail that might influence a patients' well being in an ecological situation. The clinician's goal is to find personal and adapted solutions when an individual is seeking care and help. $\mathrm{He} / \mathrm{she}$ applies what was learnt from educational courses and tailors his/her care based on the best available research.

Working in synergy to ensure better standards of care and research, scientists and clinicians could have a tremendous impact on the development of the profession. This is the vision that we share as members of the French Physiotherapy Society. The European Rehabilitation Journal will contribute to answering both scientific questions and to bringing information and context to improve clinical practice. With this new journal, we hope to be able to accompany each professional, so that he/she can continue to develop and thrive, whether he/she has an appetite for clinical practice, research or, both.

Scientific publications serve both scientists and clinicians. The latter is often involved in the generation of clinical questions depending on a specific context while the former identifies ways to evaluate those questions relying on scientific methods. Everyone has the potential to extend his or her reflection based on scientific literature. Some have the desire to go further, to confront their observations and share them with

Matthieu Guémann, French Armed Forces Biomedical Research Institute, Brétigny-sur-Orge, France mail : matthieu.guemann@intradef.gouv.fr ORCiD: 0000-0003-1896-2796 their peers by submitting their work to a scientific journal to (i) inform the community about what they have observed or discovered, (ii) get the approval and recognition of their peers and (iii) help in the growing process of knowledge in their field. This knowledge dissemination is possible because of the existence of scientific journals who, most of the time, use a peer review process that evaluates and validates (or not) the content to ensure that published scientific evidence respects certain standards and thus, could be used to improve daily practice. Because of the growing scientific corpus regarding physiotherapy and rehabilitation in France, we, the French Physiotherapy Society, have decided to create the European Rehabilitation Journal to disseminate the best available evidence in the field of rehabilitation.

As it was previously evoked in an editorial published in this journal by Elkins et al. [1], there are many advantages to the creation of a new scientific journal governed by a scientific/professional society as opposed to a publishing company. In creating the European Rehabilitation Journal, we wish to reconnect scientific publishing with its primary goal which is the production and assessment of new knowledge, avoiding the for-profit journals' influence that increasingly puts pressure on scientists with the so called "publish or perish" constraint and the race to publish in high impact factor (IF) journals [2].

In fact, as Mark Johnston brilliantly explained, the trajectory of science has been influenced by for-profit journals with high IFs because researchers carriers are more and more based on where they publish rather than what they publish [3]. At the same time, for-profit journals sometimes prefer to highlight topics that will increase their visibility rather than focusing on the quality and relevance of the article content.

Community or scientific society-based journals can really benefit authors, the society, and the entire profession by focusing on the following values:

- Peer approved and certified content. Editors of society journals are involved in their field and have high expectations about publications. Because of this involvement, maintaining the integrity of the journal is a top priority.

- Accessible and supportive. Because the editors are scientists, authors, and colleagues, they know how difficult it is to write a story that influences the field. For that reason, reviewers' comments may seem conflicting but aim to provide authors with helpful feedback. Society journals exist to serve authors and the profession.

- Agile and responsive. As editors are deeply involved in their labs, 
departments, clinical practice and institutions we use open software solutions to support and facilitate the publication process with close relationships with authors for easier discussions and modifications.

- Relevant, recognized, and targeted. As a scientific society journal, we aim to publish the most relevant content in the rehabilitation field to be recognized by our community to ensure maximum exposure for authors.

In summary, journals owned by scientific societies and run by practicing scientists represent the goals of academic publishing, focusing on selective content for the publication of research results. The editorial team have a great responsibility and put all its energy into seeking out and helping authors tell and share their stories and experiences. One can argue that it is too soon or too late to engage ourselves in such a project. We would answer that it is the best time ever regarding the professional and scientific situation of Rehabilitation science in France. Facilitating factors are the growing integration into the University system with the creation of the section 91 in the National University Council [4] which will participate in the development of University positions for Physiotherapists, Occupational therapists, Speech therapists, and Psychomotor therapists, among others. All this with the objective to increase the development of scientific reasoning for the care of patients and the community. For all these reasons, I personally encourage you as a reader, educator, scientist, professional expert to help, preserve and support academic publishing by submitting your work to European Rehabilitation Journal.

\section{References}

[1] Mark Elkins, Anne Moseley, Rafael Zambelli Pinto, et al. A new high-quality scholarly journal will help drive physiotherapy towards being an evidence-based healthcare profession in france. European Rehabilitation Journal, 1(1):1-2, 2021.

[2] Patrick D Schloss, Mark Johnston, and Arturo Casadevall. Support science by publishing in scientific society journals, 2017.

[3] Mark Johnston. Scientific society journals: By scientists for science, 2017.

[4] Décret n ${ }^{\circ}$ 2019-1107 du 30 octobre 2019 modifiant le décret no 87-31 du 20 janvier 1987 relatif au Conseil national des universités pour les disciplines médicales, odontologiques et pharmaceutiques, October 2019. 